\title{
BMJ Open Development of an English-language version of a Japanese iPad application to facilitate collaborative goal setting in rehabilitation: a Delphi study and field test
}

\author{
William Levack, ${ }^{1}$ Kounosuke Tomori, ${ }^{2}$ Kayoko Takahashi, ${ }^{3}$ Aidan J Sherrington ${ }^{4}$
}

To cite: Levack W, Tomori K, Takahashi K, et al. Development of an English-language version of a Japanese iPad application to facilitate collaborative goal setting in rehabilitation: a Delphi study and field test. BMJ Open 2018;8:e018908. doi:10.1136/ bmjopen-2017-018908

- Prepublication history and additional material for this paper are available online. To view these files, please visit the journal online (http://dx.doi. org/10.1136/bmjopen-2017018908).

Received 4 August 2017 Revised 11 January 2018 Accepted 24 January 2018

Check for updates

${ }^{1}$ Rehabilitation Teaching \& Research Unit, Department of Medicine, University of Otago, Wellington, New Zealand

${ }^{2}$ Department of Occupational Therapy, School of Health Science, Tokyo University of Technology, Yokosuka, Japan ${ }^{3}$ Department of Occupational Therapy, School of Allied Health Science, Kitasato University, Tokyo, Japan

${ }^{4}$ Department of Medicine Rehabilitation, Teaching and Research Unit, University of Otago, Dunedin, New Zealand

Correspondence to

Dr William Levack;

william.levack@otago.ac.nz

\section{ABSTRACT}

Objective This study aimed to investigate the content of an English-language version of a Japanese iPad application designed to facilitate shared decision-making around goal setting in rehabilitation: Aid for Decisionmaking in Occupational Choice-English (ADOC-E).

Design Phase 1: Delphi methods to reach consensus with an international group of expert occupational therapists on the text and images in ADOC-E. Phase 2: Testing correct recognition (unprompted and prompted) of images in ADOC-E by health service users in inpatient rehabilitation and residential care.

Setting Phase 1: International, online. Phase 2: Three healthcare services in New Zealand-(1) a residential rehabilitation service for traumatic brain injury, (2) a nursing home for frail older adults and (3) an inpatient rehabilitation ward in a public hospital.

Participants Phase 1: Fourteen experienced occupational therapists from New Zealand (4), Australia (4), UK (2) and USA (4). Phase 2: Twenty-four rehabilitation and residential care service users (10 men, 14 women; 20-95 years; Mini-Mental State Exam scores 13-30).

Results Four Delphi rounds were required to reach consensus with the experienced occupational therapists on the content of ADOC-E, ending with 100 items covering daily activities that people do and social roles they participate in. Ninety-five per cent (95/100) of ADOC-E items could each be correctly identified by over $80 \%$ of service user participants with either unprompted or prompted recognition.

Conclusion While a few of the more abstract concepts in ADOC-E (related to complex social roles) were less likely to be correctly recognised by all participants, the text and images ADOC-E were deemed to be fit for purpose overall and ready for future clinical testing.

\section{INTRODUCTION}

Collaborative goal setting is considered a key component of rehabilitation planning, with the understanding that selected goals will drive the clinical decision-making process. ${ }^{1}$ Goal setting is thought to improve teamwork practices, patient engagement in therapy
Strengths and limitations of this study

- An international group of occupational therapists from four countries were involved in the development of the content, text and images in Aid for Decisionmaking in Occupational Choice-English (ADOC-E), increasing transferability.

- Eighty per cent agreement was used as the cut-off point to decide that agreement had been reached on ADOC-E content, text and images.

- Service user testing involved collecting information on recognition of images only and did not involve input on the descriptive text and scope of content in ADOC-E.

- This study did not examine the impact of ADOC-E on health outcomes or service users experiences of rehabilitation.

and make rehabilitation more meaningful to individuals who receive these services. ${ }^{2}$ While there is currently insufficient evidence to draw strong conclusions that goal setting practices result in greater improvements in functional abilities following rehabilitation programmes, ${ }^{3}$ health professionals have an ethical obligation to involve the people receiving their services in rehabilitation planning and clinical decision-making. ${ }^{45}$ Multiple studies have demonstrated that health professionals are often not as successful at involving people with disabilities and their families in the selection of goals for therapy as they would like. ${ }^{6-13}$ Observational studies of goal setting in physiotherapy practice, ${ }^{6}$ speech-language therapy, ${ }^{78}$ paediatric rehabilitation $^{9}$ and inpatient interdisciplinary rehabilitation for adults ${ }^{1011}$ have all provided evidence of situations where health professionals dominate the goal setting process, with therapists' priorities for rehabilitation appearing to take precedence over patient 
or family preferences. This problem can be exacerbated when patients have communication or cognitive impairments, ${ }^{12}$ but these impairments are not the only barriers to person-centred communication.

One possible solution to these kinds of problems is greater use of digital technology that has been tailored to facilitate communication in rehabilitation settings. Digital technology has already created several new opportunities for augmenting traditional rehabilitation practices. For instance, iPad applications have been used as aids to increasing independence and social connectivity for people with communication ${ }^{14}$ and vision ${ }^{15}$ impairments. Various applications have also been developed and tested as therapeutic activities for people with aphasia, ${ }^{16-21}$ for children with autism spectrum disorder ${ }^{22}$ and for older adults with dementia. ${ }^{23}$ Therapists have used iPad applications to train trunk control in people with spinal cord injury, ${ }^{18}$ manual dexterity in people with neurological disorders ${ }^{19} 24-27$ and as aid to support employment success in young adults with developmental disabilities. ${ }^{28}$ However, few, if any, applications have been created to support the process of person-centred rehabilitation.

To address this issue, Tomori and colleagues developed a suite of three iPad applications in Japan to facilitate patient involvement in shared decision-making around rehabilitation planning. The first of these, the 'Aid for Decision-making in Occupation Choice' (ADOC), is an application that uses text and images to assist patients to identify meaningful activities and social roles in their lives, which could be the subject of a rehabilitation goal. ${ }^{29}$ ADOC then allows health professionals to separately identify other possible activities or social roles that they consider important in a patient's recovery and facilitates shared discussion and prioritisation of goals from these activities and roles. ${ }^{29}$ ADOC achieves greater involvement of patients in collaborative goal setting by: (1) providingmorestructurearound thegoalsettingprocess; (2) separating the patient's initial goal selection from the health professional's initial goal selection prior to more in-depth discussion of priorities; (3) presenting patients with a wide variety of possible activities and social roles to provide an indication of the breadth of possible goals they could consider and (4) using images to stimulate the patients' thinking around possible goals to consider and facilitating communication of their preferences. ${ }^{29}$

Because of the way ADOC is structured, the application encourages both health professionals and patients to consider goals of therapy that they might not otherwise identify, and it supports patients to have greater voice in the selection of goals for therapy. ADOC can also be used to produce a psychometrically valid score of a patient's degree of satisfaction with their performance before and after therapy in the identified areas of importance in their life. ${ }^{30}$ In a test of ADOC involving 116 older adults in Japan, Tomori and colleagues found that people with a Mini-Mental State Exam (MMSE) score of a low as 8 (within the range of scores for severe cognitive impairment $)^{31}$ could meaningfully participate in goal setting using ADOC. ${ }^{32}$ A second version of ADOC has been produced for children, called 'ADOC for School', and a third version is available that has a more specific focus on rehabilitation of the upper limb, called 'ADOC for Hand'. ${ }^{33}$

However, all of the ADOC products to date have been designed and tested in Japan only. We wanted to create an English-language version of ADOC (ADOC-E), but we were aware that we needed to reconsider the items in ADOC, the language used to describe them and the images used to represent them because of cultural differences between life in Japan and life in English-speaking countries. The aim of this study was to draw on occupational therapy expertise to develop a consensus around the content of ADOC-E and to test the suitability of images in ADOC-E with people with disabilities in an Englishspeaking country. Because ADOC was initially created by occupational therapists in Japan and because the content of ADOC focuses on occupation (activities people do and social roles they participate in; the primary domain of occupational therapy), we chose to concentrate on occupational therapy expertise in the initial development of ADOC-E content.

\section{METHOD}

This study involved two phases: (1) a Delphi-style method to reach a consensus among an international group of experienced occupational therapists regarding ADOC-E items, text and images and (2) testing of ADOC-E images with people in rehabilitation or nursing homes to check that the images in ADOC-E were suitably representative of the underlying concepts.

\section{Phase 1: Development of items, text and images for ADOC-E}

We aimed to recruit 12-14 occupational therapists to participate in the first phase of this study. While there is no agreement on the correct sample size of Delphi studies (or even what constitutes a 'large' or 'small' Delphi study), ${ }^{34} 12-14$ participants were considered sufficient to gain a range of opinions from people in a range of countries regarding the possible content of ADOC-E. We used purposive sampling to recruit experienced occupational therapists from four English-speaking countries (USA, UK, Australia and New Zealand) who worked in a range of clinical contexts including inpatient through to community settings, with a minimum of 5 years' work experience.

Prior to involving occupational therapy participants in this study, we translated the text for ADOC items from Japanese to English, which was checked for basic syntax and meaning by the first author (who speaks English as a first language). We also made any obvious changes that were required in the images (eg, changing an image of a person eating rice with chopsticks to an image of a person eating a steak dish with a knife and fork).

We used a Delphi-style method to progress towards consensus among the participating occupational 
therapists regarding the revised ADOC-E items, text and images. We undertook four Delphi-style rounds, using online surveys to canvas opinions on the groups' response to each ADOC-E item, text or image. In rounds 1 and 2, we sought consensus on the ADOC-E items (ie, the activity or social role they represented) and text only. In round 3, we added the ADOC-E images to the text and again sought the participants' opinions of these. In round 4 , we concentrated on the items for which consensus had not yet been reached regarding images or text. We used SurveyMonkey (an online survey application) to seek participant input in rounds 1, 2 and 4 of the study. Round 3 was conducted by e-mail as this round involved consideration of a large number of images, and it was easier for us to circulate (and for participants to comment on) these images in an Excel spreadsheet than via an online survey. In all instances, we considered consensus to be reached if over $80 \%$ of respondents reported being satisfied with an ADOC-E item, text and image. In each round, we sought feedback on how to improve each ADOC-E item, text and/or image. We collated the group's responses and feedback and shared this with all participants between each round. This included providing statistical summaries on overall agreement, plus written reports on areas of disagreement and collated summaries of recommendations for improvements. As each round of the Delphi process progressed, participants could see how ADOC items were being revised, as we progressed towards consensus, which provided additional opportunities to comment on these revisions. Any further comments of this nature were incorporated in the ongoing analysis and fed back to participants in subsequent rounds.

The research team met several times between each round to discuss the survey results and how best to address the feedback from participants. Each round was undertaken over 2 or 3 weeks, with several weeks or months between each round to analyse and summarised the feedback, plus to have any required changes to images completed by a professional illustrator.

\section{Phase 2: Testing the suitability of ADOC-E images with health service users}

Before finalising the content in the first version of ADOC-E for software development, we wanted to test the suitability of the ADOC-E images to check that these would be meaningful to potential users of the application. This was not intended as a clinical test of the function of ADOC-E but rather as a further check of the content before future clinical testing.

We aimed to recruit people from a range of different types of rehabilitation services who health professionals might normally want to involve in goal setting for rehabilitation planning. People were eligible to participate in this study if they were adults (18 years or older) and were current recipients of rehabilitation or residential care services in New Zealand. They also needed to be able to communicate with at least simple words and phrases (either verbally or via assistive technology) and score above 8 on the MMSE (the minimum level of cognitive functioning found to be required for meaningful participation in the Japanese version of ADOC) ${ }^{32}$ Participants were recruited from three separate institutions: (1) a residential rehabilitation service for traumatic brain injury, (2) a nursing home for frail older adults (3) and an inpatient rehabilitation ward in a public hospital. All participants provided written consent before contributing to the study.

As the aim of this phase of the study was to test the suitability of images prior to further investigation of ADOC-E in clinical practice (rather than to produce statistical estimates for participants' responses to images at a population level), we based sample size decision on similar studies of 'usability' in the context of survey design. ${ }^{35}$ Typically, sample sizes for usability tests are small, with a modest size of 10-15 participants being recommended, which was our target for the sample size in this phase of the study. ${ }^{35}$

For each participant, we collected basic demographic information (age, gender, ethnicity, primary diagnosis, residential status, MMSE scores). We then involved participants in an assessment of the suitability of the ADOC-E images. To check the suitability of each image, we showed the participant each image on an iPad tablet and asked three questions. We began with an open question regarding what the image was supposed to illustrate (eg, 'What do you think this is a picture of?'), that is, unprompted identification of the image. The field researcher (AS) recorded whether the participant's response was correct or incorrect. An answer was considered 'correct' if the participant's response aligned with either the general idea of the ADOC-E image or a synonym thereof. For example, 'dining' was accepted as a correct response when the ADOC-E text stated 'eating and drinking'.

To check the reliability of this method for categorising 'correct' unprompted recognition of ADOC-E images, we audio-recorded all data collection sessions and had one reviewer (WL) independently score $25 \%$ of the data. We compared agreement between the two researchers' categorisation of correct versus incorrect recognition of the ADOC-E items using Cohen's kappa coefficient.

If a participant was deemed unable to correctly identify an image, they were then asked to identify the correct response within a forced choice context, that is, a prompted identification of the image. This involved presenting the correct ADOC-E text for the image alongside the text for two other randomly chosen items within the same category, presented in a random order (eg, 'Is this a picture of a person (1) eating and drinking, (2) moving seats or (3) using the toilet?). An Excel spreadsheet was used to randomly select the two other ADOC-E items and to randomise the order in which the three items were presented in real time. A 'yes' or 'no' response was recorded for whether the participant selected the correct item out of the three options with prompting. 
A Sleeping - Getting or having a good night's sleep

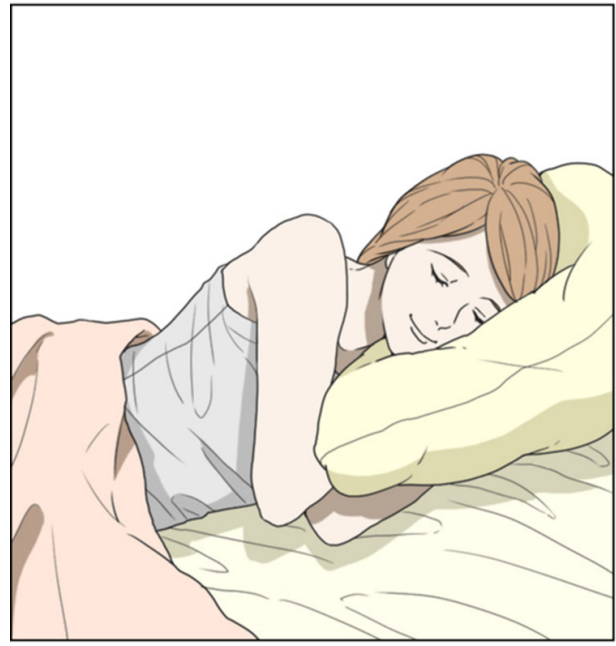

C Attending or hosting parties and social gatherings - Organising/planning events, feeling comfortable at parties, having fun in a safe way at parties

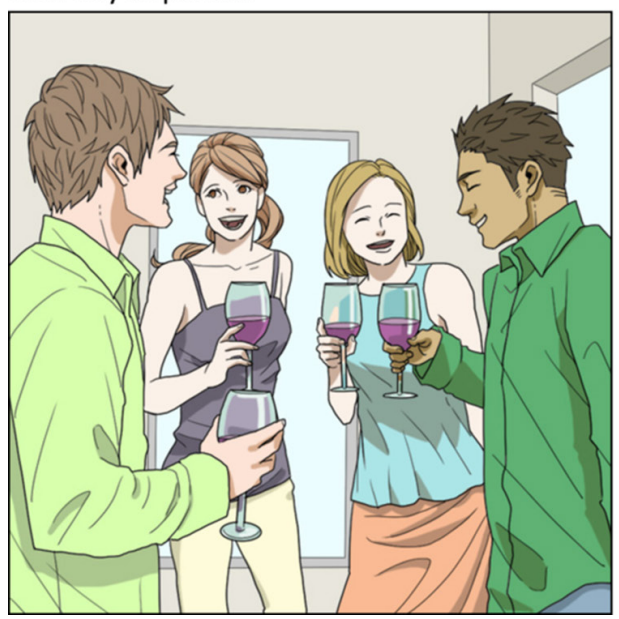

B Intimate relationships - Dating, romantic relationships, intimate/ marital relationship, sexual relationships, attracting or having a boyfriend or girlfriend

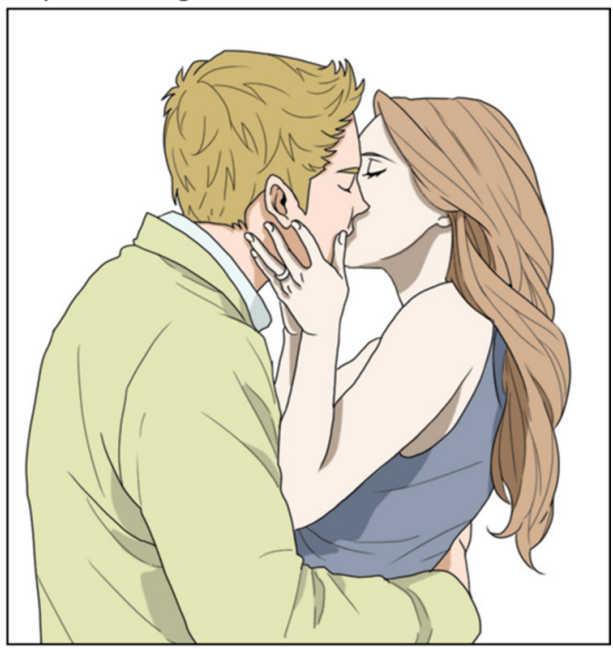

D Playing a team ball sport - Playing soccer, football, rugby, rugby league, or gridiron etc.

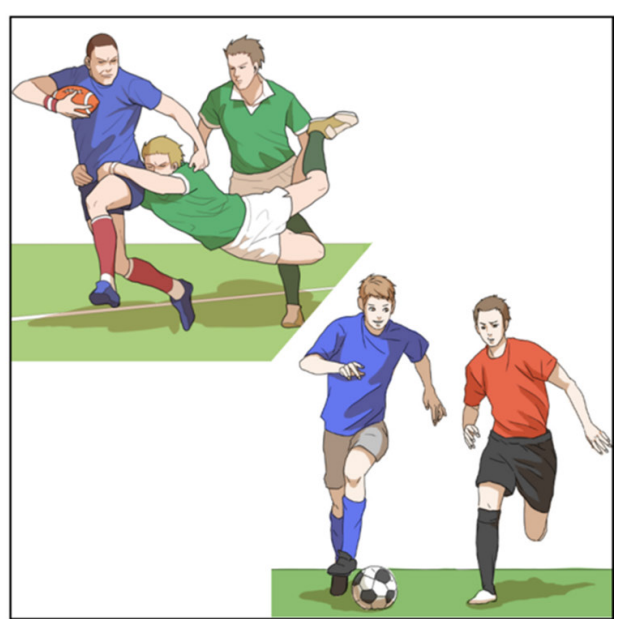

Figure 1 Provided examples of the images and text for Aid for Decision-making in Occupational Choice-English items categorised under (A) 'self-care'-sleeping; (B) 'interpersonal interactions' - intimate relationships; (C) 'social activities' attending or hosting parties and social gatherings and (D) 'sport and physical activities' playing a team ball sport.

The third question on each ADOC-E item was then asked about whether the participants considered the ADOC-E image a good representation of the intended activity or social role (ie, 'This is supposed to be a picture of someone 'preparing a meal'. Do you think that this image is a good way to show that idea?'). We recorded the participants' response dichotomously, as well as any qualitative comments they had on the ADOC-E item.

To accommodate fatigue, therapy, nursing schedules and participant preferences, we allowed participants to complete responses for as many or as few of the ADOC-E items as they wished. We also permitted individual participants to complete their data collection over multiple interviews. We limited the maximum time for a single data collection session to $90 \mathrm{~min}$ and a maximum total time involved in data collection for a single participant to $180 \mathrm{~min}$. For people who only provided responses to a proportion of the ADOC-E items, we cycled where we began data collection in the list of ADOC-E items to ensure that we had a minimum of a dozen responses to each individual image. To do this, we simply began asking each participant about the next item on the list of ADOC-E items after the last item considered by the previous participant.

Data on the participants' responses to the questions about each image were analysed with descriptive statistics. As ADOC is never used independently by people with disabilities (rather it is used as part of a conversation between health professionals and their clients or patients), we decided that correct identification of an ADOC image by $80 \%$ people with or without prompting was considered satisfactory for the purposes of the application. However, 
we examined ways we could improve any images, based on the feedback received, when less than $100 \%$ of people could correctly identify the image. All images in the original ADOC application involved a Japanese style of cartoon, which we retained in any revisions to the images in ADOC-E (see figure 1 for examples).

\section{RESULTS}

\section{Phase 1: Development of items, text and images for ADOC-E}

We recruited 14 experienced occupational therapists from four countries to participate in phase 1 of the study (four from USA, two from UK, four from Australia and four from New Zealand). The participating occupational therapists had an average of 13.3 years (SD 5.2) clinical experience. The majority $(79 \% ; 11 / 14)$ had postgraduate clinical qualifications, with two holding a doctoral degree and three further participants being doctoral candidates, with the remaining three holding bachelor degrees as their highest qualification. The occupational therapists worked in acute medical wards, specialist neurological units, mental health services, independent living facilities, universities and community rehabilitation services.

All 14 participants contributed to the first survey round. However, two participants (one from the USA and one from New Zealand) dropped out of the study after the first survey round due to other work commitments. One additional participant from the USA missed the second survey round but contributed to all three other survey rounds.

We began with 97 ADOC-E items derived from the original Japanese iPad application after removing four specially Japanese activities (eg, budo-a Japanese martial art; gètobōru-a Japanese game like croquet). These 97 ADOC-E items were divided into eight groups based on groupings in the Japanese version of ADOC: self-care (6 items); mobility (11 items); domestic life (15 items); work and learning (4 items); interpersonal interactions (5 items); social activities (7 items); sport and physical activities (20 items) and hobbies (29 items). In the first Delphi round, 98\% (95/97) of these items were considered relevant and meaningful as potential goals in rehabilitation, although suggestions to improve the scope of items or the language used to describe them were provided for $68 \%(66 / 97)$ of the ADOC-E items. The two items which did not meet our $80 \%$ agreement for inclusion at this stage were for 'baseball' (71\% agreement) and 'softball' (79\% agreement), with a few participants indicating that these were not common sports in their countries. Indeed, the whole area of 'sport and physical activity' was particularly difficult to reach consensus around, as different sports are important in different countries. Furthermore, the language used to describe sports differs from country to country, for example, the meaning of the word 'football' is highly contested. To address this problem, we decided to collapse all 'like' sports into groups represented by a single ADOC-E item, for example, 'team ball sports', 'racket and ball sports' and so on.
Another area of contention was the use of UK versus US spelling in ADOC-E. To address this problem, we revised ADOC-E to avoid these words altogether, for example, replacing the term 'radio programme' (UK spelling)/'radio program' (US spelling) with 'radio show'. Where participants from different countries disagreed on the correct term to describe certain objects or activities, we revised ADOC-E to include both sets of language, for example, referring to 'getting petrol/gas' in the ADOC-E item related to 'vehicle maintenance'.

In terms of rephrasing the text, the participants' recommendations focused primarily on clarifying the scope of each ADOC-item and on replacing clinical or technical terms with more colloquial language, for example, the ADOC category for 'mobility' was retitled 'getting around'. Following recommendations in this first Delphi round, we also added several new ADOC-E items. For instance, 'sleeping', 'relaxation' and 'dealing with emergencies' were added to the 'self-care' items. Going into phase 2 of the Delphi process, we had revised ADOC-E to include 98 items but with a few more in 'self-care' (9 items) and 'work and learning' (5 items) and a few less in 'sport and physical activity' (17 items) and 'hobbies' (28 items).

In the second Delphi round, $100 \%$ of the items were considered relevant and meaningful as potential goals in rehabilitation by all participants but with a few more minor suggestions to improve the text for 33\% (33/98) of the items. Some recommendations at this stage were to include terminology that was only relevant in one country (eg, referring to a specific social service by name that was country specific). As we intended ADOC-E to be internationally accessible, we avoided these types of changes.

'Gambling' as a hobby, which was included in the original Japanese ADOC application, was a particularly controversial ADOC-E item that provoked a lot of debate. Some questioned whether it should be included because it could be considered a vice and detrimental to the health and well-being of certain individuals. In the end, however, there was overall approval to include this item in ADOC-E for a number of reasons. Including this item reflected an acknowledgement that some people enjoyed gambling as a recreation and had a right to make their own personal choices about such activities. Participants also reflected that it was better for health professionals to know and talk about this aspect of clients' lives if it was an issue for them than for it to be a hidden problem.

Following recommendations in this second Delphi round, we added four new ADOC-E items: 'using hearing aids, glasses, contact lenses' under the 'self care' items; 'water sports—sailing, windsurfing, surfing, rowing and so on' and 'mountain sports-skiing, snowboarding, rock climbing, mountain climbing and so on' in 'sport and physical activities' and 'attending educational talks or seminars for enjoyment' under 'hobbies'. We also removed two ADOC-E items that were, after revision of the text, very similar in content to other ADOC items. 
This left us with 100 ADOC-E items for round three of the Delphi process.

In the third Delphi round, we presented the revised ADOC-E items with both their text and images. Of the 100 ADOC-E images, 91 reached our criteria for agreement by the participants, that is, $80 \%(>9 / 12)$ agreed the images were appropriate and acceptable, with 62 images being accepted by all 12 participants. Suggestions for further improvements were provided for 22 images (including the nine that did not reach our threshold of 'agreement' by the group). We revised these 22 images as recommended, and in our fourth Delphi round, canvassed the participants for their opinions on these revisions. In the fourth Delphi round, we reached our threshold for agreement, with over $80 \%(>9 / 12)$ of the participants indicating the revised ADOC-E images and text were appropriate and acceptable. All 100 agreed images were included in the next phase of our study. Raw data from each Delphi round is provided in online supplementary files $1-4$. The text from the original ADOC-E translated from Japanese and the final 100 agreed ADOC-E items are provided in online supplementary file 5 .

\section{Phase 2: Testing the suitability of ADOC-E images with health service users}

Twenty-four health service users (10 male; 14 female) from three disability and rehabilitation organisations took part in phase 2 of this study. Their mean MMSE score was 25, with a range of 13-30, indicative of severe to no cognitive impairment. ${ }^{31}$ All participants were above the MMSE score cut-off for using ADOC (see table 1). ${ }^{32}$ The median number of ADOC-E items that each participant commented on was 52 items (IQR: 66.25 items; range: 11-100 items). Eight participants commented on all 100 ADOC-E items. Each individual ADOC-E item was considered by 13-16 different people.

Cohen's kappa, as a measure of agreement between the two researchers categorising the participants' open

\begin{tabular}{|c|c|}
\hline Gender & 10 men, 14 women. \\
\hline Age & $\begin{array}{l}\text { Mean: } 57.2 \text { years }(S D 23.1) \text {; median: } \\
61 \text { years; range: } 20-95 \text { years; } 10 \text { under } \\
50 \text { years; } 14 \text { over } 50 \text { years. }\end{array}$ \\
\hline NZ European & 20 NZ European; 4 Māori. \\
\hline Health condition & $\begin{array}{l}5 \text { stroke, } 10 \text { traumatic brain injury, } 6 \text { frail } \\
\text { elderly ( } 3 \text { with mild to no dementia; } 3 \\
\text { with moderate dementia), } 1 \text { Guillain-Barré } \\
\text { syndrome, } 1 \text { functional limb weakness, } 1 \\
\text { mobility impairment postsurgery. }\end{array}$ \\
\hline MMSE & Mean: 25.3 (SD 5.4); range: 13-30. \\
\hline $\begin{array}{l}\text { Residential } \\
\text { status }\end{array}$ & $\begin{array}{l}14 \text { short-term residential rehabilitation; } 10 \\
\text { permanent residential care. }\end{array}$ \\
\hline
\end{tabular}

MMSE, minimental state exam; NZ, New Zealand. recognition of the ADOC-E items, was 0.77 , indicative of substantial agreement. Where the two researchers disagreed, the field researcher tended to have been more conservative in his judgement regarding open recognition (ie, more likely to consider a response non-recognition than the second researcher). In our reporting of participants' responses below, we have retained the more conservative scoring of image recognition.

Overall, 40 of the 100 ADOC-E images were correctly identified by the participants without prompting (ie, open recognition) all of the time. Correct recognition of 72 of the 100 ADOC-E images was achieved by all participants when prompted recognition was also an option. Ninety-five of the 100 ADOC-E images were correctly identified by $80 \%$ of participants when both unprompted and prompted recognition was counted. Five ADOC-E images could not be correctly identified even with prompting for $20 \%-40 \%(3-6 / 15)$ of participants. The images which proved most difficult for participants to identify tended to be those related to social participation. Specifically, these five difficult ADOC-E items were for 'paid employment'-represented by a person working on a computer in an office; 'voluntary (unpaid) work'-represented by a person picking up rubbish in a park; 'higher education/vocational training' - represented by a picture of a university student studying books; 'public interactions'-represented by a person getting direction from a stranger on the street and 'using public services'-represented by a person getting a book out of a library. These difficult ADOC-E items represented more abstract concepts and more individualised social roles than more easily recognisable ones such as, for instance, images representing 'using the toilet', 'shopping' or 'playing golf'.

For 19 of the 100 ADOC-E images, over $80 \%$ of the participants thought the picture did not accurately represent the intended concept. Frequently, in these cases, the participants focused on the specific nature of the picture rather than the broader concept we wanted it to represent. Again this was more of a problem for the more abstract concepts. So, for instance, while 100\% of participants (14/14) correctly identified a picture of people going to the cinema, $50 \%$ of people did not consider this a good representation of the broader ADOC item 'enjoying arts-going to shows/concert/art galleries/movies/museum' because it was just a representation of one of these activities. In fact, 9 of these 19 images were considered problematic by participants even though they were correctly identified, to the satisfaction of the researchers, by $100 \%$ of the participants without prompting. The participants also provided useful feedback on confusing or distracting aspects of imagessuch as pictures of people in cars who were not wearing seatbelts-that could be easily corrected. See online supplementary file 6 for the raw data from this phase of the study. 


\section{DISCUSSION}

This paper provides a report on the scientific work that has led to the development of a prototype of an English-language version of a Japanese iPad application designed to facilitate shared decision-making around goal setting in rehabilitation. Overall, the Delphi process resulted in 100 ADOC-E items, each represented by text and images that were agreed on by an international group of experienced occupational therapists as potentially relevant foci for goal setting in many different areas of rehabilitation practice. The majority of the ADOC-E images could be identified correctly by rehabilitation or residential care service users (including those with mild to severe cognitive impairment) when prompting was provided. Given that ADOC-E is not intended to completely replace communication with patients but rather to supplement it, we believe that this level of recognition means the ADOC-E images are currently fit for purpose-although in future work, we aim to continue to refine and improve the content of the application. Indeed, evidence from studies from the Japanese version of ADOC has demonstrated that we can expect ADOC-E to facilitate more person-centred goal setting in rehabilitation contexts. ${ }^{29} 32$

This study has also usefully highlighted the limitations of the current ADOC-E items, which need to be considered when this application is used in clinical practice. The ADOC-E items which were most easily recognised by participants were those related to physical activities. More abstract concepts relating to social roles that people engage in tended to be harder to represent pictorially and therefore harder (though not impossible) for participants to identify. Users of ADOC-E may need to talk more explicitly about these ADOC-E items when using the application in clinical practice.

One additional limitation of using single images to represent the ADOC-E items, highlighted by the occupational therapy participants, is that age, gender and ethnicity of the person in the image did not necessarily match that of the people using ADOC-E. An alternative here would be to redesign ADOC-E so that application creates an avatar for each user, matched on basic demographic characteristics, which is then used in all the images for that person. This however would require a substantial reprogramming of the application, so would need to be considered in future work. Another area for improvement of ADOC-E would be the addition of more content to increase specificity of individual items. For instance, the ADOC-E item related to 'enjoying arts' could be split into several separate ADOC items representing 'going to shows/concerts', 'visiting art galleries', 'going to the movies' and 'visiting museums'. The patient participants in this study also made a number of suggestions regarding possible improvements to the ADOC-E images, but we have not yet acted on all of these suggestions or taken them back to the participants for reconsideration.

One strength of this study was that we engaged with occupational therapists from four different countries in the initial revision of the ADOC-E items. This has increased the likelihood that ADOC-E will be applicable in a variety of English-speaking countries. However, we have only tested the ADOC-E images with health service users in one country (New Zealand) and predominantly with people who have neurological or age-related impairments. We have also only sought input from a relatively small number of occupational therapists and patients to date. We do not yet know how relevant ADOC-E might be to the work of other members of the multidisciplinary team. We also do not yet know how these images might be received by health service users in other English-speaking countries or by people with more diverse types of health conditions. Future research should examine the actual application of ADOC-E in English-speaking clinical settings to test whether ADOC-E changes the way different health professionals and patients interact with one another during rehabilitation planning or therapeutic activities. It would also be useful to gather information about the perspectives of health professionals other than occupational therapists on the use, content and scope of items and images in ADOC-E and on its potential for application to clinical practice.

Past research has indicated that health professionals struggle to involve patients in shared decision-making around goal setting to the extent that they think is desirable. ${ }^{6-911}$ This lack of involvement is partly due to patients being relatively passive when it comes to asserting what the goals of their rehabilitation should be ${ }^{7}$ or a tendency to acquiesce to the goals of therapists. ${ }^{10}$ ADOC-E has the potential to address these problems by presenting patients with a wide range of life areas in which they might like to consider setting goals and permitted patients to consider these to be potentially relevant targets for therapy.

\section{CONCLUSION}

Experienced occupational therapists have been involved in the construction of content of an English-language version of a Japanese iPad application, ADOC-E, designed to facilitate shared decision-making around goal setting for rehabilitation. Testing of ADOC-E images with health service users has indicated that the majority of these are a fair representation of the concept they are intended to represent and fit for purpose in terms of achieving the objectives of the ADOC-E application. Future research needs to trial ADOC-E in clinical practice to examine its impact on person-centred rehabilitation processes and outcomes.

Acknowledgements We would like to thank the healthcare service users and organisation involved in this project, including the patients, residents and staff at Capital \& Coast District Health Board, ABI Rehabilitation and Sprott House.

Contributors WMML drafted the manuscript and led the design and implementation of both phases of the study. He collected all data and completed the initial data analysis for all stages of phase 1 . He recruited the rehabilitation and disability support services involved in phase 2 . He provided oversight for the analysis of phase 2 . He led the responses to peer review feedback on the draft manuscript. KTo designed the ADOC application and translated the original ADOC 
content from Japanese to English. He contributed to the design of both phase 1 and phase 2 of the study. He arranged all revisions to illustrations in ADOC arising from the study. He contributed to analysis of all study data, writing of the manuscript, responses to peer reviewers and read and approved the final manuscript. KTa contributed to the design of both phase 1 and phase 2 of the study. She contributed to analysis of all study data, writing of the manuscript, responses to peer reviewers and read and approved the final manuscript. She also facilitated communication between Japanese and English. AJS contributed to the design of phase 2 of the study. He recruited all participants, collected all data and completed the initial data analysis for phase 2 . He wrote a report on the outcome of phase 2 of the study that informed the first draft of this manuscript. He contributed to the writing of the pape and read and approved the final manuscript.

Funding No funding was involved in phase 1. Funding for data collection in phase 2 of this study was provided by the University of Otago Research Committee. ADOC research in Japan is supported by Kakenhi Grant from the Japanese Society for the Promotion of Science (Grant number: 15K01425).

Competing interests None declared.

Patient consent Obtained.

Ethics approval All participants were informed about the aim of the study and that all participation was voluntary. All participants provided written informed consent before contributing to the study. Ethics approval was provided by the University of Otago's Human Ethics Committee (Health) (H16/086).

Provenance and peer review Not commissioned; externally peer reviewed.

Data sharing statement Raw data from this study are available in the supplementary files.

Open Access This is an Open Access article distributed in accordance with the Creative Commons Attribution Non Commercial (CC BY-NC 4.0) license, which permits others to distribute, remix, adapt, build upon this work non-commercially, and license their derivative works on different terms, provided the original work is properly cited and the use is non-commercial. See: http://creativecommons.org/ licenses/by-nc/4.0/

(C) Article author(s) (or their employer(s) unless otherwise stated in the text of the article) 2018. All rights reserved. No commercial use is permitted unless otherwise expressly granted.

\section{REFERENCES}

1. Levack WMM, Dean S. Processes in rehabilitation. In: Dean S, Siegert R, Taylor W, eds. Interprofessional rehabilitation: a personcentred approach. Sussex: John Wiley \& Sons, Ltd, 2012:79-108.

2. Levack WM, Dean SG, Siegert RJ, et al. Purposes and mechanisms of goal planning in rehabilitation: the need for a critical distinction. Disabil Rehabil 2006;28:741-9.

3. Levack WM, Weatherall M, Hay-Smith EJ, et al. Goal setting and strategies to enhance goal pursuit for adults with acquired disability participating in rehabilitation. Cochrane Database Syst Rev 2015:CD009727.

4. Levack WMM, Siegert RJ, Pickering N. Ethics and goal setting. In: Siegert RJ, Levack WMM, eds. Rehabilitation goal setting: theory, practice, and evidence. Boca Raton: CRC Press, 2015:67-87.

5. Haas J. Ethical considerations of goal setting for patient care in rehabilitation medicine. Am J Phys Med Rehabil 1993;72:228-32.

6. Baker SM, Marshak HH, Rice GT, et al. Patient participation in physical therapy goal setting. Phys Ther 2001;81:1118-26.

7. Rohde A, Townley-O'Neill K, Trendall K, et al. A comparison of client and therapist goals for people with aphasia: a qualitative exploratory study. Aphasiology 2012;26:1298-315.

8. Berg K, Askim T, Balandin S, et al. Experiences of participation in goal setting for people with stroke-induced aphasia in Norway. A qualitative study. Disabil Rehabil 2017;39:1122-30

9. Nijhuis BJ, Reinders-Messelink HA, de Blécourt AC, et al. Goal setting in Dutch paediatric rehabilitation. Are the needs and principal problems of children with cerebral palsy integrated into their rehabilitation goals? Clin Rehabil 2008;22:348-63.

10. Barnard RA, Cruice MN, Playford ED. Strategies used in the pursuit of achievability during goal setting in rehabilitation. Qual Health Res 2010;20:239-50.
11. Levack WM, Dean SG, Siegert RJ, et al. Navigating patient-centered goal setting in inpatient stroke rehabilitation: how clinicians control the process to meet perceived professional responsibilities. Patient Educ Couns 2011;85:206-13.

12. Playford ED, Dawson L, Limbert V, et al. Goal-setting in rehabilitation: report of a workshop to explore professionals' perceptions of goalsetting. Clin Rehabil 2000;14:491-6.

13. Conneeley AL. Interdisciplinary collaborative goal planning in a post-acute neurological setting: a qualitative study. Br J Occup Ther 2004;67:248-55

14. Bradshaw J. The use of augmentative and alternative communication apps for the iPad, iPod and iPhone: an overview of recent developments. Tizard Learning Disability Review 2013;18:31-7.

15. Mednick Z, Jaidka A, Nesdole R, et al. Assessing the iPad as a tool for low-vision rehabilitation. Can J Ophthalmol 2017;52:13-19.

16. Des Roches CA, Balachandran I, Ascenso EM, et al. Effectiveness of an impairment-based individualized rehabilitation program using an iPad-based software platform. Front Hum Neurosci 2014:8:1015

17. McNaughton $\mathrm{D}$, Light J. The iPad and mobile technology revolution: benefits and challenges for individuals who require augmentative and alternative communication. Augment Altern Commun 2013;29:107-16.

18. Fizzotti G, Rognoni C, Imarisio A, et al. Tablet technology for rehabilitation after spinal cord injury: a proof-of-concept. Stud Health Technol Inform 2015;210:479-83.

19. White J, Janssen $H$, Jordan $L$, et al. Tablet technology during stroke recovery: a survivor's perspective. Disabil Rehabil 2015;37:1186-92.

20. Kurland J, Wilkins AR, Stokes P. iPractice: piloting the effectiveness of a tablet-based home practice program in aphasia treatment. Semin Speech Lang 2014;35:51-63.

21. Ramsberger G, Messamer P. Best practices for incorporating non-aphasia-specific apps into therapy. Semin Speech Lang 2014;35:17-24.

22. Yan F. A sunny day: Ann and ron's world an ipad application for children with autism. Serious games development and applications. 2011:129-38.

23. Leng FY, Yeo D, George S, et al. Comparison of iPad applications with traditional activities using person-centred care approach: impact on well-being for persons with dementia. Dementia 2014;13:265-73.

24. Saposnik G, Chow C-M, Gladstone D, et al. iPad technology for home rehabilitation after stroke (iHOME): a proof-of-concept randomized trial. International Journal of Stroke 2014;9:956-62.

25. Kizony R, Zeilig G, Dudkiewicz I, et al. Tablet apps and dexterity: comparison between 3 age groups and proof of concept for stroke rehabilitation. J Neurol Phys Ther 2016;40:31-9.

26. Rand D, Zeilig G, Kizony R. Rehab-let: touchscreen tablet for selftraining impaired dexterity post stroke: study protocol for a pilot randomized controlled trial. Trials 2015;16:277.

27. Saposnik G, Chow CM, Gladstone D, et al. iPad technology for home rehabilitation after stroke (iHOME): a proof-of-concept randomized trial. Int J Stroke 2014;9:956-62.

28. Hill DA, Belcher L, Brigman HE, et al. The apple iPad (TM) as an innovative employment support for young adults with autism spectrum disorder and other developmental disabilities. Journal of Applied Rehabilitation Counseling 2013;44:28.

29. Tomori K, Uezu S, Kinjo S, et al. Utilization of the iPad application: aid for decision-making in occupation choice. Occup Ther Int 2012;19:88-97.

30. Tomori K, Saito $\mathrm{Y}$, Nagayama $\mathrm{H}$, et al. Reliability and validity of individualized satisfaction score in aid for decision-making in occupation choice. Disabil Rehabil 2013;35:113-7.

31. Tombaugh TN, Mclntyre NJ. The mini-mental state examination: a comprehensive review. J Am Geriatr Soc 1992;40:922-35.

32. Tomori K, Nagayama H, Saito $\mathrm{Y}$, et al. Examination of a cut-off score to express the meaningful activity of people with dementia using iPad application (ADOC). Disabil Rehabil Assist Technol 2015;10:126-31.

33. Ohno K, Tomori K, Takebayashi T, et al. Development of a tool to facilitate real life activity retraining in hand and arm therapy. $\mathrm{Br} J$ Occup Ther 2017;80:310-8.

34. Akins RB, Tolson H, Cole BR. Stability of response characteristics of a Delphi panel: application of bootstrap data expansion. BMC Med Res Methodol 2005;5:37.

35. Blair J, Conrad FG. Sample size for cognitive interview pretesting. Public Opin Q 2011;75:636-58. 